\title{
Improve Microwave Quantum Illumination Via Optical Parametric Amplifier
}

\author{
Biao Xiong, Xun Li, Xiao-Yu Wang, Ling Zhou ${ }^{1}$ \\ School of Physics, Dalian University of Technology, Dalian 116024, People's Republic of \\ China
}

\begin{abstract}
Quantum illumination is a quantum-optical sensing technique in which an entangled source is exploited to improve the detection of a low-reflectivity object that is immersed in a bright thermal background. Entangled sources between microwave and optical fields can be exploited to improve detection in microwave quantum illumination technique. We proposed a scheme to enhance the entanglement between the output fields of microwave and optical cavity by introducing optical parametric amplifier medium in cavity optomechanical system. We show that improving signal-to-noise ratio and decreasing error probability of detection can be obtained consequently even for objects with low reflectivity in the presence of optical parametric amplifier.
\end{abstract}

Keywords: Microwave quantum illumination, Optical parametric amplifier, Lower error probability

\section{Introduction}

The concept of quantum illumination was first put forward by S. Lloyd [1], where one of a pair of entangled photons is sent into a target region, the other photon is retained at the receiver; a joint measurement between the reflected back from target region and the retained photon can discriminate whether the object presents or not. The quantum illumination is of great significance. By the technique, we can defeat passive eavesdropping [2]. Compared with traditional radar, quantum illumination has the characteristics of higher signal-to-noise ratio and the lower error probability [3-5] . The singlephoton quantum illumination [1] was extended into Gaussian-state quantum illumination very soon [6 - 8 ]. Based on [1] and [6] schemes, E. D. Lopaeva et. 
al. experimentally realized the quantum illumination protocol at the optical wave lengths [9].

On the other hand, optomechanical interaction can couple high-frequency optical field with low-frequency field with nonlinear interaction, which offer us many amazing potential application such as entangling macroscopic and microscopic object [10 16, 38] , performing high-precision measurements [17 19], processing quantum information [20-22] and cooling a mechanical oscillator to its quantum ground state [23, 24], producing sideband comb [25]. The entanglement between optical field and microwave field provides us the possibility to detect invisible object. Most recently, S. Barzanjeh et al. theoretically extended optical quantum illumination to microwave quantum illumination by using an electro-optomechanical (EOM) converter where the optomechanical system capacitively couples with LC oscillating circuit through the movement of the mechanical oscillator driven by the optical driven fields [26]. In Shabir Barzanjeh's quantum-illumination protocol, microwaves produced by the LC circuit and emitted to the target area can entangle the optical waves which are retained to operate a jointed measurement with the reflected signal. They showed that microwave quantum illumination dramatically outperforms a conventional (coherent state) microwave radar of the same transmitted energy, achieving an orders-of-magnitude lower detection-error probability, which is related to the entanglement degree. In optomechanical system, there are many ways to improve entanglement [2731], one of which is to put an optical parametric amplifier (OPA) crystal in an optomechanical system [32].

Inspired by the ideas elaborated in Refs. [6 8, 26, 32], we put forward a scheme to improve the signal-to-noise ratio of microwave quantum illumination by introducing $\chi^{(2)}$ nonlinear medium. We show that the entanglement between optical field and the microwave field can be enhanced because of the enlarged effective optomechanical coupling strength, meanwhile the signal-to-noise ratio is improved and the error probability of detection can be decreased.

\section{Model and the Hamiltonian of the system}

The EOM system is shown in Fig:1(a), a mechanical resonator (MR) capacitively couples to the microwave cavity (MC) consisting of LC circuit with resonant frequency $\omega_{w}$ on the one side, and on the other side couples to the optical cavity (OC) with resonant frequency $\omega_{o}$ in which lied a $\chi^{(2)}$ 


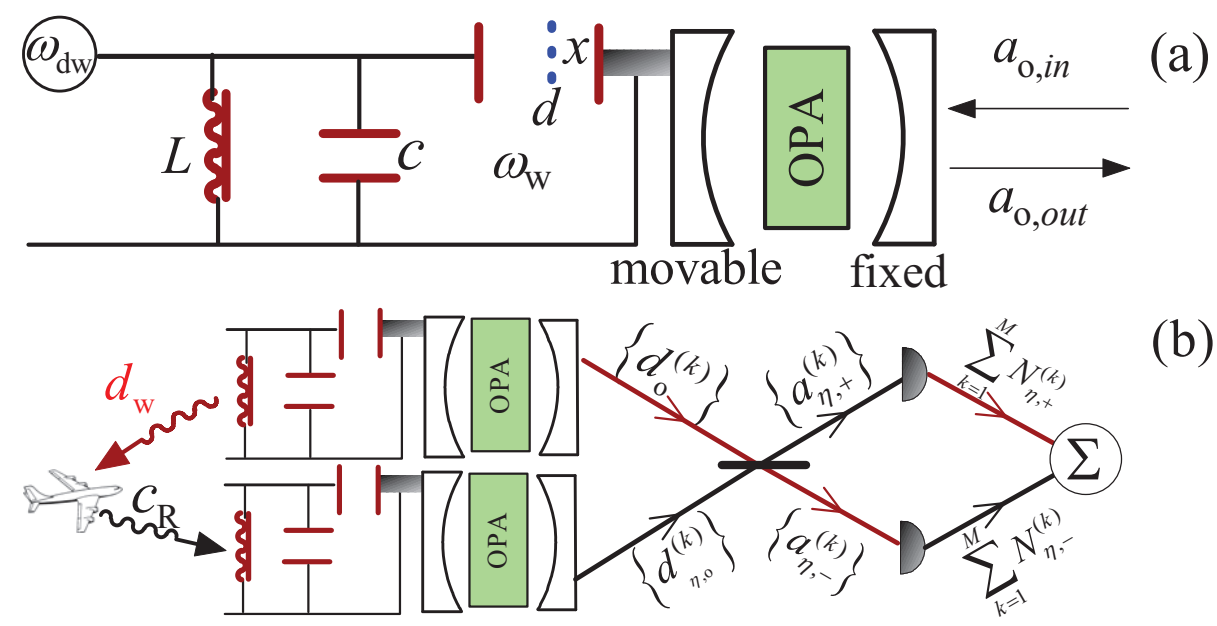

Figure 1: (a): Schematic of the EOM system. The microwave cavity and the optical cavity contained an OPA crystal can be coupled simultaneously with mechanical oscillator. (b): Schematic of quantum illumination, which is composed of two EOM system, one of which is used as the transmitter to transmit the entangled microwave and optical idler fields, and the other as a receiver, which receives the reflected microwave, and it's optical output is used in a joint measurement with the retained idler.

nonlinear medium. The microwave quantum illumination device as shown in Fig.1(b) contains two identical EOM system, one of which is used as the transmitter to transmit the microwaves and the idle optical waves, and the other as a receiver, which receives the reflected microwaves, and can output optical waves [26]. For convenience, we define them as EOM transmitters and EOM receivers, respectively. The Hamiltonian of the hybrid system in the interaction picture reads [26, 33 38]

$H=\hbar \omega_{m} b^{\dagger} b+\hbar \sum_{j=w, o}\left[\Delta_{j}-g_{j}\left(b+b^{\dagger}\right)\right] a_{j}^{\dagger} a_{j}+i \hbar E_{j}\left(a_{j}^{\dagger}-a_{j}\right)+i \hbar G\left(e^{i \theta} a_{o}^{\dagger 2}-e^{-i \theta} a_{o}^{2}\right)$,

where $a_{j}(b)$ is the annihilation operator of cavity field (mechanical resonator) with frequency $\omega_{j}\left(\omega_{m}\right) . \Delta_{j}=\omega_{j}-\omega_{d, j}$ denotes the detuning of the two cavities frequencies $\omega_{j}$ from their driving field frequencies $\omega_{d, j}$, with $j=$ $w, o$ describing the microwave cavity and optical cavity, respectively. $g_{o}=$ $\frac{\omega_{o}}{l} \sqrt{\frac{\hbar}{2 m \omega_{m}}}$ gives the OC-MR coupling rate, with $m$ the effective mass of MR, and $l$ the length of the optical cavity, while the MC-MR coupling $g_{w}=$ $\frac{\mu \omega_{w}}{2 d} \sqrt{\frac{\hbar}{2 m \omega_{m}}}$ is corresponding to the effective mass $m$ of MR and a separation $d$ 
of the two plates marked in Fig.1(a), and $\mu=C /\left(C+C_{0}\right)$ is the dimensionless parameter, with $C$ being the capacitance of parallel capacitor, and $C_{0}$ being the bare capacitance corresponding to the $d$ separation of the two palates. The driving strength of cavity $E_{j}=\sqrt{2 P_{j} \kappa_{j} / \hbar \omega_{d, j}}$ with the power $P_{j}$. And $\kappa_{j}$ describes the losing rates of cavity $j . G$ is the nonlinear gain of the OPA, and $\theta$ is the phase of the field driving the OPA. Considering the loss rates of the cavities and resonator, we can give the nonlinear Heisenberg-Langevin equation

$$
\begin{aligned}
\dot{a}_{w} & =\left(-i \Delta_{w}-\kappa_{w}\right) a_{w}+i g_{w}\left(b+b^{\dagger}\right) a_{w}+E_{w}+\sqrt{2 \kappa_{w}} a_{w, i n} \\
\dot{a}_{o} & =\left(-i \Delta_{o}-\kappa_{o}\right) a_{o}+i g_{o}\left(b+b^{\dagger}\right) a_{o}+E_{o}+2 G e^{i \theta} a_{o}^{\dagger}+\sqrt{2 \kappa_{o}} a_{o, i n}, \\
\dot{b} & =\left(-i \omega_{m}-\gamma_{m}\right) b+i g_{o} a_{o}^{\dagger} a_{o}+i g_{w} a_{w}^{\dagger} a_{w}+\sqrt{2 \gamma_{m}} b_{i n}
\end{aligned}
$$

where $a_{w, i n}, a_{o, \text { in }}$ and $b_{i n}$ are the input noise operators of the microwave, of the optical and of the mechanical resonator, with the following correlation functions

$$
\begin{aligned}
\left\langle a_{j, i n}(t) a_{j, i n}^{\dagger}\left(t^{\prime}\right)\right\rangle & =\left(\bar{n}_{j}^{T}+1\right) \delta\left(t-t^{\prime}\right), \\
\left\langle b_{i n}(t) b_{i n}^{\dagger}\left(t^{\prime}\right)\right\rangle & =\left(\bar{n}_{b}^{T}+1\right) \delta\left(t-t^{\prime}\right),
\end{aligned}
$$

where $\bar{n}_{j}^{T}=\left[\exp \left(\hbar \omega_{j} / k_{B} T\right)-1\right]^{-1}$ is the equilibrium mean thermal photon numbers of the optical $(j=o)$ and microwave $(j=w)$ fields. And $\bar{n}_{b}^{T}=\left[\exp \left(\hbar \omega_{m} / k_{B} T\right)-1\right]^{-1}$ is the mean thermal excitation numbers of the resonator. We can safely assume $\bar{n}_{o}^{T} \approx 0$ since $\hbar \omega_{j} / k_{B} T \gg 1$ at optical frequencies and low temperatures, while thermal microwave photons and thermal excitation phonons of resonator cannot be neglected usually, even at very low temperatures.

We can expand the operator of the optical field as its steady-state mean value and a small fluctuation with zero mean value under the strong driving condition, i.e. $a_{j} \rightarrow \alpha_{j}+a_{j}$. By setting the derivatives of $\alpha_{j}$ to zero, we obtain the steady-state mean values $\alpha_{j}$ as

$$
\begin{aligned}
\alpha_{w} & =\frac{E_{w}}{i \Delta_{w}+\kappa_{w}}, \\
\alpha_{o} & =\frac{\left(-i \Delta_{o}+\kappa_{o}+2 G e^{i \theta}\right) E_{o}}{\kappa_{o}^{2}+\Delta_{o}^{2}-4 G^{2}} .
\end{aligned}
$$

Accordingly, the linearized Langevin equations for fluctuation operators be- 
come

$$
\begin{aligned}
\dot{a}_{w} & =\left(-i \Delta_{w}-\kappa_{w}\right) a_{w}+i g_{w}^{\prime}\left(b+b^{\dagger}\right)+\sqrt{2 \kappa_{w}} a_{w, i n} \\
\dot{a}_{o} & =\left(-i \Delta_{o}-\kappa_{o}\right) a_{o}+i g_{o}^{\prime}\left(b+b^{\dagger}\right)+2 G e^{i \theta} a_{o}^{\dagger}+\sqrt{2 \kappa_{o}} a_{o, i n} \\
\dot{b} & =\left(-i \omega_{m}-\gamma_{m}\right) b+i g_{o}^{\prime} a_{o}^{\dagger}+i g_{o}^{\prime *} a_{o}+i g_{w}^{\prime} a_{w}^{\dagger}+i g_{w}^{\prime *} a_{w}+\sqrt{2 \gamma_{m}} b_{i n}
\end{aligned}
$$

where $g_{j}^{\prime}=\alpha_{j} g_{j}(j=w, o)$ is the effective coupling rate. We rewrite Eqs. (9)(11) and the corresponding transposed conjugate in the compact form

$$
\dot{f}=\Lambda f+\zeta,
$$

where $f=\left(a_{w}, a_{w}^{\dagger}, a_{o}, a_{o}^{\dagger}, b, b^{\dagger}\right)^{T}, \zeta=\left(\sqrt{2 \kappa_{w}} a_{w, i n}, \sqrt{2 \kappa_{w}} a_{w, i n}^{\dagger}, \sqrt{2 \kappa_{o}} a_{o, i n}\right.$, $\left.\sqrt{2 \kappa_{o}} a_{o, i n}^{\dagger}, \sqrt{2 \gamma_{m}} b_{i n}, \sqrt{2 \gamma_{m}} b_{i n}^{\dagger}\right)^{T}$ where the superscript $T$ means transposition, and the coefficient matrix $\Lambda$ is given by

$$
\left[\begin{array}{cccccc}
-i \Delta_{w}-\kappa_{w} & 0 & 0 & 0 & i g_{w}^{\prime} & i g_{w}^{\prime} \\
0 & i \Delta_{w}-\kappa_{w} & 0 & 0 & -i g_{w}^{\prime \star} & -i g_{w}^{\prime \star} \\
0 & 0 & -i \Delta_{o}-\kappa_{o} & 2 G e^{i \theta} & i g_{o}^{\prime} & i g_{o}^{\prime} \\
0 & 0 & 2 G e^{-i \theta} & i \Delta_{o}-\kappa_{o} & -i g_{o}^{\prime \star} & -i g_{o}^{\prime \star} \\
i g_{w}^{\prime \star} & i g_{w}^{\prime} & i g_{o}^{\prime \star} & i g_{o}^{\prime} & -i \omega_{m}-\gamma_{m} & 0 \\
-i g_{w}^{\prime \star} & -i g_{w}^{\prime} & -i g_{o}^{\prime \star} & -i g_{o}^{\prime} & 0 & i \omega_{m}-\gamma_{m}
\end{array}\right]
$$

The system is stable only if all of the eigenvalues of $\Lambda$ are of real negative parts, which means Routh-Hurwitz criterion [39] satisfied. Because of the matrix $\Lambda$ is $6 \times 6$ dimensions, so it is difficult to obtain the analytical solution of steady condition. We assume that $\Delta_{w}=-\Delta_{o}=-\omega_{m}$, i.e., the optical field satisfies blue-sideband condition while the microwave field is in the redsideband. We numerically solve the eigenvalues of $\Lambda$ to ensure the stability of our system, which is shown in Fig. 2. It is clearly shows that the system can reach its steady state at a wide range of $G$ and $\theta$, although the system contains a nonlinear medium with amplification effect. Hereafter, we will choose parameters within steady region.

We can easily solve Eqs. (9)-(11) in the frequency domain by Fourier transform $O(\omega)=\frac{1}{\sqrt{2 \pi}} \int d t O(t) e^{i \omega t}$. By substituting the solutions of Eqs. (99)(11) in frequency domain into the standard input-output relation $d_{j}=a_{j, \text { out }}=$ 
$\sqrt{2 \kappa} a_{j}-a_{j, i n}$, we have

$$
\begin{aligned}
d_{w}(\omega)= & A_{1}(\omega) a_{w, i n}(\omega)+A_{2}(\omega) a_{w, i n}^{\dagger}(\omega)+A_{3}(\omega) a_{o, i n}(\omega) \\
& +A_{4}(\omega) a_{o, i n}^{\dagger}(\omega)+A_{5}(\omega) b_{i n}(\omega)+A_{6}(\omega) b_{i n}^{\dagger}(\omega), \\
d_{o}(\omega)= & B_{1}(\omega) a_{w, i n}(\omega)+B_{2}(\omega) a_{w, i n}^{\dagger}(\omega)+B_{3}(\omega) a_{o, i n}(\omega) \\
& +B_{4}(\omega) a_{o, i n}^{\dagger}(\omega)+B_{5}(\omega) b_{i n}(\omega)+B_{6}(\omega) b_{i n}^{\dagger}(\omega),
\end{aligned}
$$

where the coefficients $A_{j}(\omega)$ and $B_{j}(\omega)(j=1,2 \ldots 6)$ are given in Appendix A (A.1 A.15). We see clearly that $d_{w}(\omega)$ is contributed partly by optical field (contain $a_{o, \text { in }}(\omega)$ and $a_{o, i n}^{\dagger}(\omega)$ ), similarly, $d_{o}(\omega)$ is contributed partly by microwave field (contain $a_{w, i n}(\omega)$ and $a_{w, i n}^{\dagger}(\omega)$ ), which exhibits that there is quantum correlation between the optical field and microwave field. The correlation functions of Eq. (5) and (6) are transformed to the frequency domain as

$$
\begin{aligned}
\left\langle a_{j, i n}(\omega) a_{j, i n}^{\dagger}\left(\omega^{\prime}\right)\right\rangle & =\left(\bar{n}_{j}^{T}+1\right) \delta\left(\omega+\omega^{\prime}\right), \\
\left\langle b_{i n}(\omega) b_{i n}^{\dagger}\left(\omega^{\prime}\right)\right\rangle & =\left(\bar{n}_{b}^{T}+1\right) \delta\left(\omega+\omega^{\prime}\right) .
\end{aligned}
$$

We define $n(o \mid w)$ to describe the mean number of microwave fields contributed by the output optical fields. Similarly, $n(w \mid o)$ describes the mean number of photons contributed by the output microwave fields. From Eqs. (14) and (15), we have

$$
\begin{aligned}
& n(o \mid w)=\left(\left|B_{1}(\omega)\right|^{2}+\left|B_{2}(\omega)\right|^{2}\right) \bar{n}_{w}^{T}+\left|B_{2}(\omega)\right|^{2}, \\
& n(w \mid o)=\left(\left|A_{3}(\omega)\right|^{2}+\left|A_{4}(\omega)\right|^{2}\right) \bar{n}_{o}^{T}+\left|A_{4}(\omega)\right|^{2} .
\end{aligned}
$$

\section{MICROWAVE-OPTICAL OUTPUT ENTANGLEMENT}

Now we introduce the covariance matrix (CM) $V$ in the frequency domain. The matrix element of the $4 \times 4 \mathrm{CM}$ can be expressed as

$$
\delta\left(\omega+\omega^{\prime}\right) V_{i j}(\omega)=\frac{1}{2}\left\langle u_{i}(\omega) u_{j}\left(\omega^{\prime}\right)+u_{j}\left(\omega^{\prime}\right) u_{i}(\omega)\right\rangle
$$

where

$$
\mathbf{u}(\omega)=\left[X_{w}(\omega), Y_{w}(\omega), X_{o}(\omega), Y_{o}(\omega)\right]^{T}
$$




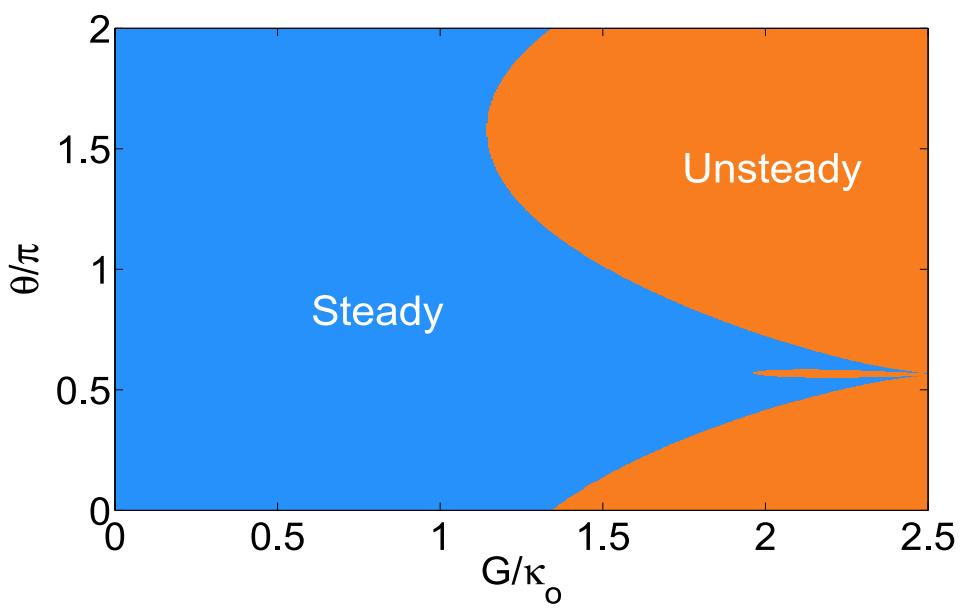

Figure 2: The stability of the system affected by $G / \kappa_{o}$ and $\theta / 2 \pi$, where $T=30 \mathrm{mk}, \lambda=$ $1064 \mathrm{~nm}, \omega_{m} / 2 \pi=10 \mathrm{MHz}, Q=\omega_{m} / \gamma_{m}=30 \times 10^{3}, \omega_{w} / 2 \pi=10 \mathrm{GHz}, g_{\mathrm{w}} / 2 \pi=0.327$, $g_{\mathrm{o}} / 2 \pi=115.512, \Delta_{\mathrm{w}}=-\omega_{m}, \Delta_{\mathrm{o}}=\omega_{m}, \kappa_{\mathrm{w}}=0.24 \omega_{m}, \kappa_{\mathrm{o}}=0.2 \omega_{m}, P_{\mathrm{o}}=10 P_{\mathrm{w}}=10 \mathrm{~mW}$.

and $X_{j}(\omega)=\left(d_{j}(\omega)+d_{j}^{\dagger}(\omega)\right) / \sqrt{2}, Y_{j}(\omega)=\left(d_{j}(\omega)-d_{j}^{\dagger}(\omega)\right) / i \sqrt{2}$ with $j=$ $o, w$ denoting the quadratures fluctuation of the optical and microwave field, respectively.

With the help of CM, we can easily quantify the output entanglement of the microwave fields and optical fields using logarithmic negativity [40], i.e.,

$$
E_{N}=\max \left\{0,-\ln 2 \eta^{-}\right\}
$$

where $\eta^{-}=2^{-1 / 2}\left[\Sigma(V)-\sqrt{\Sigma(V)^{2}-4 \operatorname{det} V}\right]^{1 / 2}$ is the lowest symplectic eigenvalue of the partial transpose of the $\mathrm{CM}$, and $\Sigma(V)=\operatorname{det} A+\operatorname{det} B-$ $2 \operatorname{det} C$, with the $A, B, C$ being the $2 \times 2$ matrix, taking from the $\mathrm{CM}$

$$
V=\left(\begin{array}{cc}
A & C \\
C^{T} & B
\end{array}\right)
$$

We plot the output entanglement between the cavity field and microwave field in Fig.3. We also keep all of the parameters meeting the steady state condition that displayed in Fig.2. As shown in Fig.3(a), the logarithmic negativity $E_{N}$ is increased with the increasing of the value of the nonlinear gain $G$ around $\theta=0.62 \pi$. Fig. 3 (b) also exhibits that the maximum value 

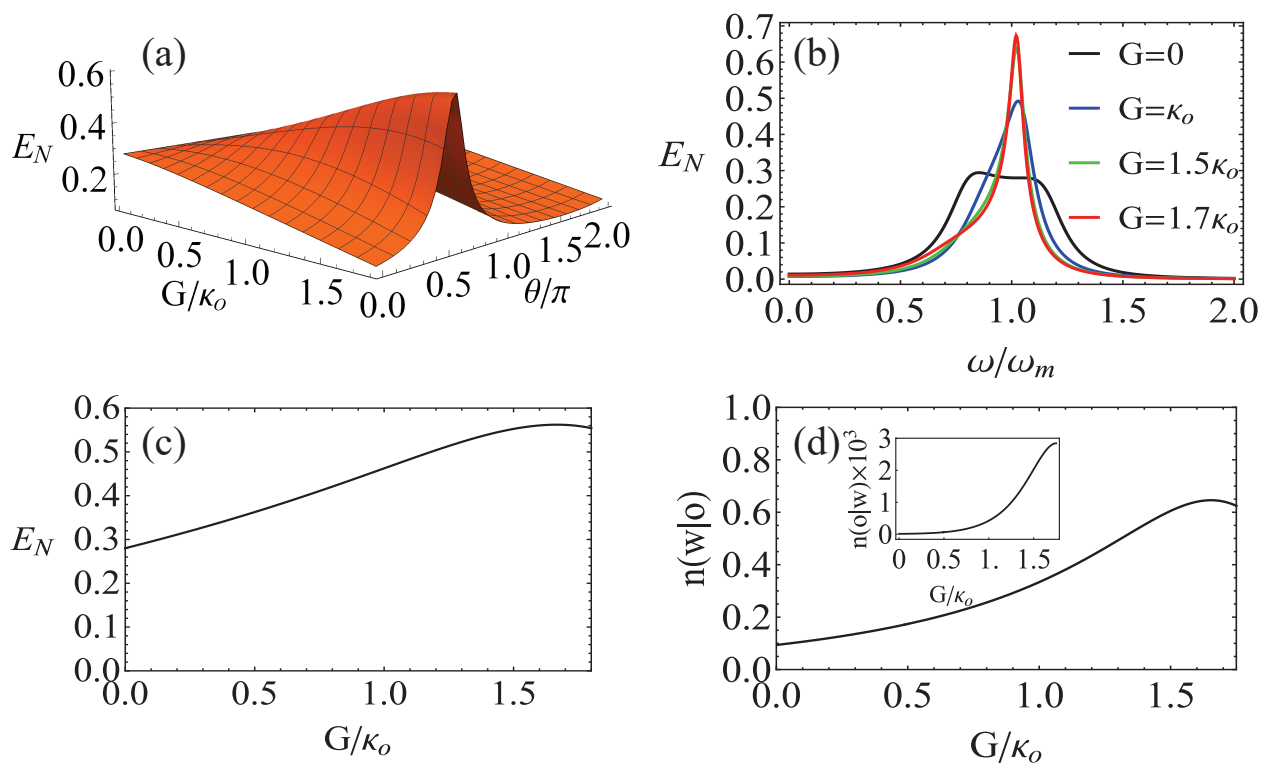

Figure 3: (a) $E_{N}$ versus $G / \kappa_{o}$ and $\theta / 2 \pi$, (b) $E_{N}$ versus $\omega / \omega_{m}$, (c) $E_{N}$ versus $G / \kappa_{o}$, and (d) $n(o \mid w)\left(n(w \mid o)\right.$ in subgraph) versus $G / \kappa_{o}$. We set $\omega=\omega_{m}$ for (a),(c),(d) and $\theta=0.62 \pi$ for (b), (c),(d). The other parameters are the same as in Fig,2

of $E_{N}$ with nonzero value of $G$ is obviously larger than that with $G=0$, meanwhile, with the increasing of $G$, the maximum value of entanglement is enhanced. From the definition of $n(o \mid w)$ and $n(w \mid o)$, we know that they exhibit quantum correlation and should have relation with entanglement. Eq. (18) and (19) is visualized in Fig.3 (d) and its subgraph. We see that $n(o \mid w)$ and $n(w \mid o)$ increase with the increasing of $G$ which is consistent with Fig $3(\mathrm{c})$.

In order to understand the effect of OPA medium on the entanglement between the cavity field and microwave field, we perform a squeezing transformation [41 43]

$$
a_{o}=\cosh (r) \widetilde{a}_{o}-i e^{i \theta} \sinh (r) \widetilde{a}_{o}^{\dagger},
$$

where $r=\frac{1}{4} \ln \frac{\Delta_{o}+2 G}{\Delta_{o}-2 G}$. Then the Hamiltonian can be written as

$$
\begin{aligned}
H= & \hbar \omega_{m} b^{\dagger} b+\hbar \Delta_{o}^{\prime} \widetilde{a}_{o}^{\dagger} \widetilde{a}_{o}-\hbar g_{o s} \widetilde{a}_{o}^{\dagger} \widetilde{a}_{o}\left(b+b^{\dagger}\right)+\hbar g_{o p}\left(e^{-i\left(\theta+\frac{\pi}{2}\right)} \widetilde{a}_{o}^{2}+e^{i\left(\theta+\frac{\pi}{2}\right)} \widetilde{a}_{o}^{\dagger 2}\right) \\
& \left(b+b^{\dagger}\right)+\hbar \Delta_{w} a_{w}^{\dagger} a_{w}-\hbar g_{w} a_{w}^{\dagger} a_{w}\left(b+b^{\dagger}\right)+H_{d r i}^{\prime},
\end{aligned}
$$


with

$$
H_{d r i}^{\prime}=i \hbar E_{w}\left(a_{w}^{\dagger}-a_{w}\right)+i \hbar E_{o}\left[\left(\cosh (r)+e^{i\left(\theta+\frac{\pi}{2}\right)} \sinh (r)\right) \widetilde{a}_{o}^{\dagger}-h . c .\right],
$$

where $\Delta_{O}^{\prime}=\Delta_{o} \cosh (2 r)-2 G \sinh (2 r), g_{o s}=\frac{g_{o} \Delta_{o}}{\sqrt{\Delta_{o}^{2}-4 G^{2}}}, g_{o p}=\frac{g_{o} G}{\sqrt{\Delta_{o}^{2}-4 G^{2}}}$. We can see that the third and fourth terms in Eq. (25) describe the standard optomechanical radiation-pressure and parametric amplification interactions, respectively, with the controllable strengths $g_{o s}$ and $g_{o p}$. The strength of the optomechanical radiation-pressure $g_{o s}$ is enhanced than $g_{o}$, i.e., $g_{o s} \succ g_{o}$. The entanglement between the cavity field and microwave field is resulted from the optomechanical radiation-pressure interactions via a common bus of mechanical oscillator; therefore, the entanglement can be enhanced by the participating of $\chi^{(2)}$ medium. The forth term also should contribute to the squeezing of the optical mode.

\section{ERROR PROBABILITY FOR DETECTION}

As shown in Fig.1b, the microwave emitted by the EOM transmitter is shined on the surface of the target object, the reflected back signal by the object is the input signal to the EOM receiver. The optical output of the receiver, similar with Eq.(15), can be written as

$$
\begin{aligned}
d_{\eta, o}(\omega)= & B_{1}(\omega) a_{R}(\omega)+B_{2}(\omega) a_{R}^{\dagger}(\omega)+B_{3}(\omega) a_{o, i n}(\omega) \\
& +B_{4}(\omega) a_{o, i n}^{\dagger}(\omega)+B_{5}(\omega) b_{i n}(\omega)+B_{6}(\omega) b_{i n}^{\dagger}(\omega),
\end{aligned}
$$

where we have defined a new input operator of microwave cavity $a_{R}=a_{B}$ under the hypothesis that the target region do not contain a low-reflectivity object (hypothesis $H_{0}$ ), and $a_{R}=\sqrt{\eta} d_{w}+\sqrt{1-\eta} a_{B}$ under the hypothesis that the target region contain a low-reflectivity object (hypothesis $H_{1}$ ). Here $a_{B}$ is the annihilation operator of the back ground noise which is in a thermal state with the photon number $n_{B}$ with hypothesis $H_{0}$ and $n_{B} /(1-\eta)$ with hypothesis $H_{1}$. We can safely assume $\left\langle a_{B}^{\dagger} a_{B}\right\rangle=n_{B}$ for both hypothesis, since the effective reflectivity (including propagation losses and target reflectivity) $\eta$ is small and $n_{B}$ is very large.

As shown in Fig.1(b), the returned optical signal is combined with the retained idler on a 50-50 beam splitter whose output is

$$
a_{\eta, \pm}=\frac{d_{\eta, o} \pm d_{o}}{\sqrt{2}} .
$$


(a)
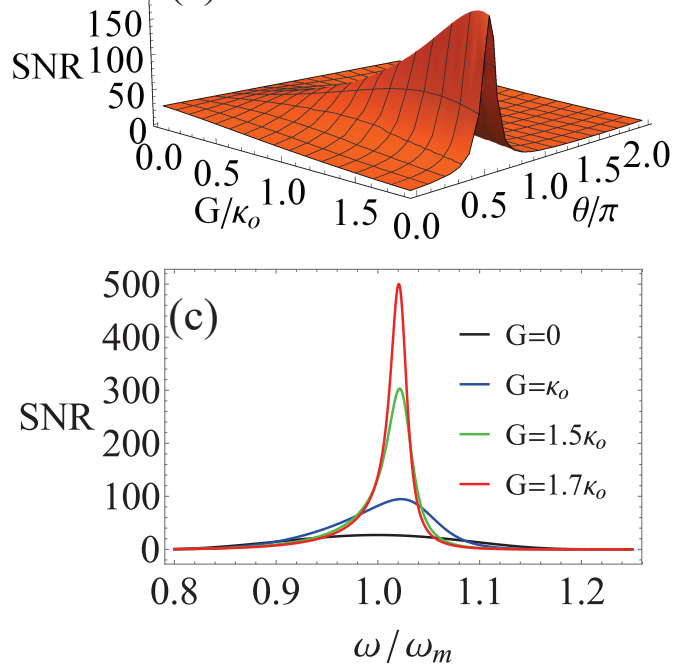

(b)
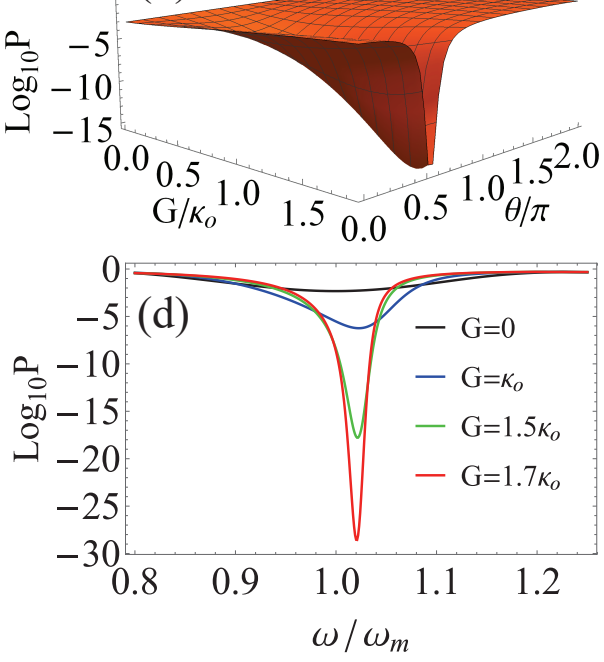

Figure 4: Plot of: (a) signal-to-noise ratio SNR and (b) error probability $\mathrm{P}$ as a function of $G$ and $\theta$. (c) signal-to-noise ratio SNR and (d) error probability P versus $\omega / \omega_{m}$. We set $\eta=0.07, M=10^{6}, n_{B}=610$ and the other parameters are the same as in Fig. 2 .

For quantum illumination target detection, our signal-idler mode pair analysis must be extended to a continuous-wave EOM system whose $W_{m}$-Hzbandwidth output fields are used in a $t_{m}$-sec-duration measurement involving $M=t_{m} W_{m} \gg 1$ independent, identically-distributed mode pairs to discriminate target absence from target presence [44]. The identically-distribute mode pairs are then photo-detected. Finally, the target absence-or-presence decision is made by comparing the difference of the two detectors' total photon counts

$$
N_{\eta}=\sum_{k=1}^{M}\left(N_{\eta,+}^{(k)}-N_{\eta,-}^{(k)}\right),
$$

where $N_{\eta, \pm}^{(k)}=a_{\eta, \pm}^{(k) \dagger} a_{\eta, \pm}^{(k)}$ is corresponding to the photon-counts. The error probability of quantum illumination can be expressed as [26]

$$
\mathrm{P}=\frac{\operatorname{erfc}(\mathrm{SNR} / 8)}{2}
$$



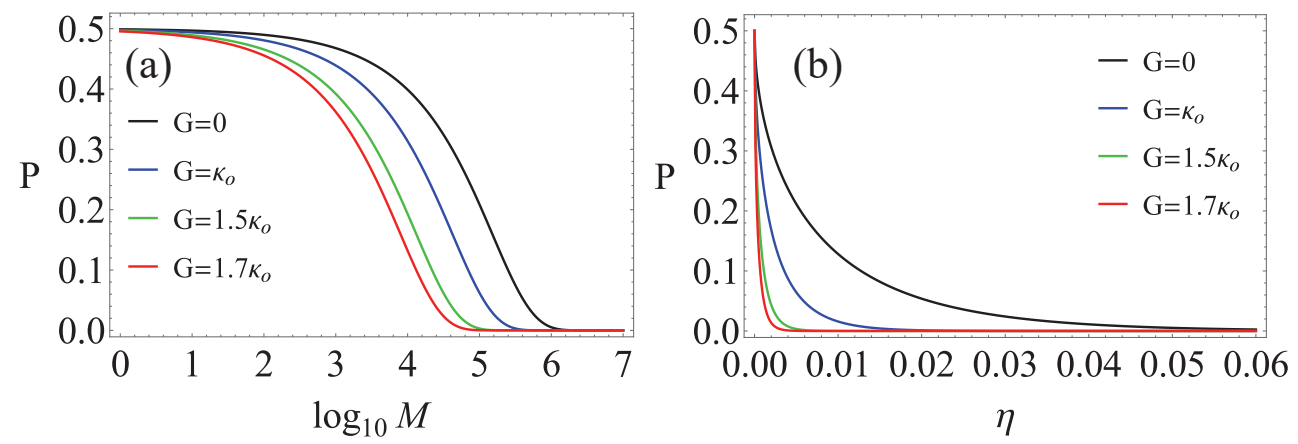

Figure 5: Plot of error probability $\mathrm{P}$ versus (a) mode pairs $M$ and (b) efficient reflectivity $\eta$. We set $\eta=0.05$ for (a), $M=10^{6}$ for (b). $\theta=0.62 \pi$ and $\omega=1.02 \omega_{m}, n_{B}=610$ for both . The other parameters see Fig. 2 .

where the $M$-Mode signal-to-noise ratio is

$$
\mathrm{SNR}=\frac{4 M\left[\left(\left\langle N_{\eta,+}\right\rangle_{H_{1}}-\left\langle N_{\eta,-}\right\rangle_{H_{1}}\right)-\left(\left\langle N_{\eta,+}\right\rangle_{H_{0}}-\left\langle N_{\eta,-}\right\rangle_{H_{0}}\right)\right]^{2}}{\left(\sqrt{\left\langle\left(\Delta N_{\eta,+}-\Delta N_{\eta,-}\right)^{2}\right\rangle_{H_{0}}}+\sqrt{\left\langle\left(\Delta N_{\eta,+}-\Delta N_{\eta,-}\right)^{2}\right\rangle_{H_{1}}}\right)^{2}} .
$$

Eqs .(29) (30) can be calculated easily, since we have calculated $\left\langle N_{\eta}\right\rangle_{H_{j}}$ and $\left\langle\left(\Delta N_{\eta,+}-\Delta N_{\eta,-}\right)^{2}\right\rangle_{H_{j}}$ in Appendix B (B.1,B.22).

Now we discuss the properties of quantum illumination. We numerically simulate the signal-to-noise ratio SNR and error probability $\mathrm{P}$ as a function of $G$ and $\theta$ or $\omega / \omega_{m}$, which is shown in Fig 4(a), Fig,4(b), Fig.4(c) and Fig 4(d) respectively. The maximum value of signal-to-noise ratio SNR with OPA is higher than that without OPA [see Figs.4(a) and 4(c)], and the error probability $\mathrm{P}$ with OPA is decreased comparing with that of without OPA. Moreover, the larger values of $G$, the higher value of SNR and lower value of error probability P. Jointly considering Fig 4 with Fig. 3 , we see that the larger the entanglement, the higher the signal-to-noise ratio SNR, and the lower the error probability of detection. That is to say, when we can archive ideal entanglement in the region in terms of the parameters $G, \theta$ and $\omega / \omega_{m}$, the signal-to-noise ratio SNR also obtain its maximum values with the minimum error probability $\mathrm{P}$.

In order to further study the advantages of our microwave quantum illumination system, we plot error probability $\mathrm{P}$ versus identically-distributed mode pairs $M$ or efficient reflectivity rate $\eta$ at different OPA gain coefficient $G$, where we set $\eta=0.05$ for Fig 5 (a) and $M=10^{6}$ for Fig 5 (b). From the 

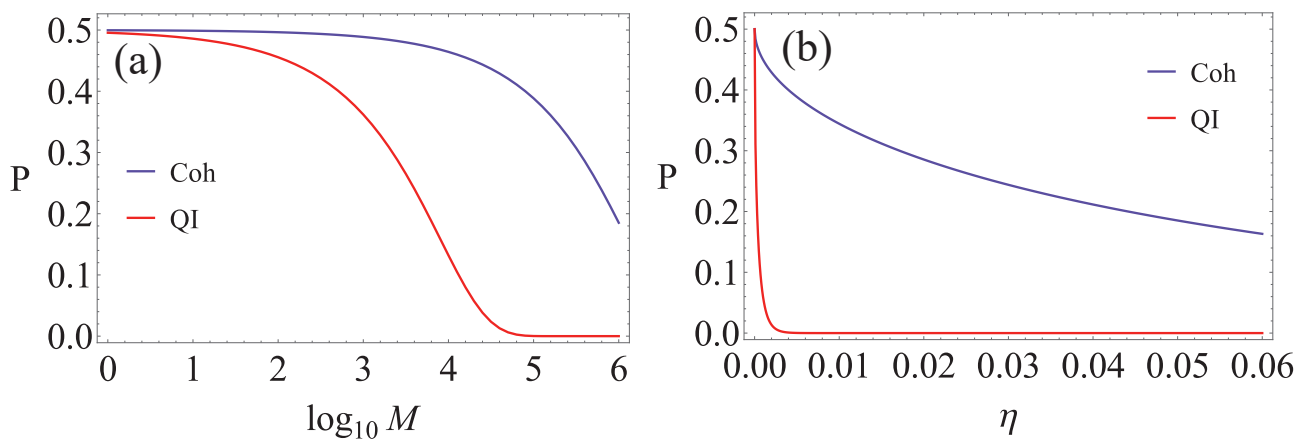

Figure 6: The error probability $\mathrm{P}$ versus (a) mode pairs $M$ and (b) efficient reflectivity $\eta$. We set $\eta=0.05$ for (a), $M=10^{6}$ for (b). For both of (a) and (b), $\theta=0.62 \pi, \omega=1.02 \omega_{m}$, and $n_{B}=610$. The other parameters are the same with Fig. [3.

simulation results shown in Fig. 5 , we can see that the superiority is obvious (lower error probability) in the presence of OPA. Fig 5 (a) shows that for certain value of $M$, the error probability $\mathrm{P}$ decreases with the increasing of values $G$, and the more identically-distributed mode pairs $M$, the lower error probability P. Furthermore, $P$ can reach almost zero when $M=10^{6}$ at $G=1.7 \kappa_{o}$. Fig.5(b) shows that without the OPA medium, the error probability $\mathrm{P}$ decreases with the increase of the efficient reflectivity $\eta$, but it still keep higher value (see the black-line). With the help of OPA medium, the error probability $\mathrm{P}$ can be decreased greatly. Even for the low efficient reflectivity object, the error probability $P$ still can achieve very low value with the assistance of OPA medium, which is of great importance for practical applications.

We compare our quantum illumination system with the conventional radar, whose error probability [7] is

$$
\mathrm{P}_{\mathrm{coh}}=\frac{\operatorname{erfc}\left(\mathrm{SNR}_{\mathrm{coh}} / 8\right)}{2},
$$

where

$$
\mathrm{SNR}_{\mathrm{coh}}=\frac{4 \eta M n_{w}}{2 n_{B}+1},
$$

here $n_{w}=\left\langle d_{w}^{\dagger} d_{w}\right\rangle=\left(\left|A_{1}(\omega)\right|^{2}+\left|A_{2}(\omega)\right|^{2}\right) \bar{n}_{w}^{T}+\left(\left|A_{3}(\omega)\right|^{2}+\left|A_{4}(\omega)\right|^{2}\right) \bar{n}_{o}^{T}+$ $\left(\left|A_{5}(\omega)\right|^{2}+\left|A_{6}(\omega)\right|^{2}\right) \bar{n}_{b}^{T}+\left|A_{2}(\omega)\right|^{2}+\left|A_{4}(\omega)\right|^{2}+\left|A_{6}(\omega)\right|^{2}$. We plot the error 
probability of our quantum illumination and conventional radar as a function of $M$ and $\eta$ in Fig. 6(a) and Fig. 6(b), which shows that our quantum illumination is distinct superior to traditional radar. Here we set $G=1.7 \kappa_{o}$.

\section{Summary}

In this paper, by introducing OPA medium, we show that the entanglement between optical field and microwave field can be improved. We analyse the mechanism that the OPA nonlinear medium affects the quantum entanglement, meanwhile our investigation exhibits that the signal-to-noise ratio of the detected object with low reflectivity rate $\eta$ can be improved, and the the error probability of the detection can be reduced by introducing the OPA nonlinear medium. That may give us effective way to improve microwave quantum illumination. Since the OPA nonlinear medium has been widely used in experiment and is mature technique, the presented scheme should be applicable.

\section{ACKNOWLEDGEMENT}

We would like to thank Mr. Wen-Zhao Zhang and Jiong Cheng for helpful discussions. This work was supported by the NSF of China under Grant numbers 11474044 . 


\section{Appendix A. The expression of output fields}

The coefficients $A_{j}(\omega)$ and $B_{j}(\omega)$ of Eqs. (14), (15) can be expressed as

$$
\begin{aligned}
& A_{1}(\omega)=\frac{1}{u} \frac{4 i \omega_{m}\left|g_{w}^{\prime}\right|^{2} \kappa_{w}}{\Delta_{w}^{-2}}-\frac{2 \kappa_{w}}{\Delta_{w}^{-}}-1, \\
& A_{2}(\omega)=\frac{1}{u} \frac{4 i \omega_{m} g_{w}^{\prime 2} \kappa_{w}}{\Delta_{w}^{-} \Delta_{w}^{+}}, \\
& A_{3}(\omega)=-\frac{1}{u} \frac{4 i \omega_{m} \sqrt{\kappa_{w} \kappa_{o}}\left(2 G e^{-i \theta} g_{o}^{\prime} g_{w}^{\prime}-\Delta_{o}^{+} g_{o}^{\prime *} g_{w}^{\prime}\right)}{\Delta_{w}^{-}\left(\Delta_{o}^{-} \Delta_{o}^{+}-4 G^{2}\right)}, \\
& A_{4}(\omega)=-\frac{1}{u} \frac{4 i \omega_{m} \sqrt{\kappa_{w} \kappa_{o}}\left(2 G e^{i \theta} g_{o}^{\prime *} g_{w}^{\prime}-\Delta_{o}^{-} g_{o}^{\prime} g_{w}^{\prime}\right)}{\Delta_{w}^{-}\left(\Delta_{o}^{-} \Delta_{o}^{+}-4 G^{2}\right)}, \\
& A_{5}(\omega)=\frac{1}{u} \frac{2 i g_{w}^{\prime} \sqrt{\kappa_{w} \gamma_{m}} \Delta_{m}^{+}}{\Delta_{w}^{-}} \\
& A_{6}(\omega)=\frac{1}{u} \frac{2 i g_{w}^{\prime} \sqrt{\kappa_{w} \gamma_{m}} \Delta_{m}^{-}}{\Delta_{w}^{-}} \\
& B_{1}(\omega)=\frac{1}{u} \frac{4 i \omega_{m} \sqrt{\kappa_{o} \kappa_{w}}\left(2 G e^{i \theta} g_{o}^{\prime *} g_{w}^{\prime *}+\Delta_{o}^{+} g_{o}^{\prime} g_{w}^{\prime *}\right)}{\Delta_{w}^{-}\left(\Delta_{o}^{-} \Delta_{o}^{+}-4 G^{2}\right)}, \\
& B_{2}(\omega)=\frac{1}{u} \frac{4 i \omega_{m} \sqrt{\kappa_{o} \kappa_{w}}\left(2 G e^{i \theta} g_{o}^{\prime *} g_{w}^{\prime}+\Delta_{o}^{+} g_{o}^{\prime} g_{w}^{\prime}\right)}{\Delta_{w}^{+}\left(\Delta_{o}^{-} \Delta_{o}^{+}-4 G^{2}\right)}, \\
& B_{3}(\omega)=-\frac{1}{u} \frac{4 i \omega_{m} \kappa_{o}\left(2 G e^{i \theta} g_{o}^{\prime *}+\Delta_{o}^{+} g_{o}^{\prime}\right)\left(2 G e^{-i \theta} g_{o}^{\prime}-\Delta_{o}^{+} g_{o}^{\prime *}\right)}{\left(\Delta_{o}^{-} \Delta_{o}^{+}-4 G^{2}\right)^{2}} \\
& -\frac{2 \kappa_{o} \Delta_{o}^{+}}{\Delta_{o}^{-} \Delta_{o}^{+}-4 G^{2}}-1 \text {, } \\
& B_{4}(\omega)=-\frac{1}{u} \frac{4 i \omega_{m} \kappa_{o}\left(2 G e^{\mathrm{i} \theta} g_{o}^{\prime *}+\Delta_{o}^{+} g_{o}^{\prime}\right)\left(2 G e^{\mathrm{i} \theta} g_{o}^{\prime *}-\Delta_{o}^{-} g_{o}^{\prime}\right)}{\left(\Delta_{o}^{-} \Delta_{o}^{+}-4 G^{2}\right)^{2}} \\
& +\frac{4 \kappa_{o} G e^{\mathrm{i} \theta}}{\Delta_{o}^{-} \Delta_{o}^{+}-4 G^{2}} \\
& B_{5}(\omega)=\frac{1}{u} \frac{2 i \sqrt{\kappa_{o} \gamma_{m}} \Delta_{m}^{+}\left(2 G e^{\mathrm{i} \theta} g_{o}^{*}+\Delta_{o}^{+} g_{o}^{\prime}\right)}{\Delta_{o}^{-} \Delta_{o}^{+}-4 G^{2}}, \\
& B_{6}(\omega)=\frac{1}{u} \frac{2 i \sqrt{\kappa_{o} \gamma_{m}} \Delta_{m}^{-}\left(2 G e^{\mathrm{i} \theta} g_{o}^{\prime *}+\Delta_{o}^{+} g_{o}^{\prime}\right)}{\Delta_{o}^{-} \Delta_{o}^{+}-4 G^{2}}, \\
& \Delta_{j}^{ \pm}=i\left(\omega \pm \Delta_{j}\right)-\kappa_{j}, j=w, o \\
& \Delta_{m}^{ \pm}=i\left(\omega \pm \omega_{m}\right)-\gamma_{m},
\end{aligned}
$$




$$
u=-\frac{2 i \omega_{m}\left[2 G\left(g_{o}^{\prime 2} e^{-\mathrm{i} \theta}-g_{o}^{\prime * 2} e^{\mathrm{i} \theta}\right)+2 i \Delta_{o}\left|g_{o}^{\prime}\right|^{2}\right]}{\Delta_{o}^{-} \Delta_{o}^{+}-4 G^{2}}-\frac{4 \omega_{m} \Delta_{\mathrm{w}}\left|g_{\mathrm{w}}^{\prime}\right|^{2}}{\Delta_{\mathrm{w}}^{-} \Delta_{\mathrm{w}}^{+}}+\Delta_{m}^{-} \Delta_{m}^{+} .
$$

\section{Appendix B. The calculation of Signal-to-noise ratio} follows

In order to calculate Eq. (29) and (30), firstly we calculate $\left\langle N_{\eta, \pm}\right\rangle_{H_{j}}$ as

$$
\begin{aligned}
\left\langle N_{\eta, \pm}\right\rangle_{H_{1}} & =\frac{1}{2}\left(D_{1} \bar{n}_{w}^{T}+D_{2} \bar{n}_{o}^{T}+D_{3} \bar{n}_{b}^{T}+D_{4} \bar{n}_{B}^{T}+F_{1}+F_{2}+F_{3}+F_{4}\right),(B) \\
\left\langle N_{\eta, \pm}\right\rangle_{H_{0}} & =\left\langle N_{\eta, \pm}\right\rangle_{H_{1}}(\eta \rightarrow 0)
\end{aligned}
$$

where the coefficients of Eq.(B.1) is

$$
\begin{aligned}
D_{1}= & {\left[\sqrt{\eta} B_{2}^{*}(\omega) A_{1}(-\omega)+\sqrt{\eta} B_{1}^{*}(\omega) A_{2}^{*}(\omega) \pm B_{2}^{*}(\omega)\right] } \\
& \times\left[\sqrt{\eta} B_{1}(\omega) A_{2}(\omega)+\sqrt{\eta} B_{2}(\omega) A_{1}^{*}(-\omega) \pm B_{2}(\omega)\right] \\
& +\left[\sqrt{\eta} B_{2}^{*}(\omega) A_{2}(-\omega)+\sqrt{\eta} B_{1}^{*}(\omega) A_{1}^{*}(\omega) \pm B_{1}^{*}(\omega)\right] \\
& \times\left[\sqrt{\eta} B_{1}(\omega) A_{1}(\omega)+\sqrt{\eta} B_{2}(\omega) A_{2}^{*}(-\omega) \pm B_{1}(\omega)\right], \\
D_{2}= & {\left[\sqrt{\eta} B_{2}^{*}(\omega) A_{3}(-\omega)+\sqrt{\eta} B_{1}^{*}(\omega) A_{4}^{*}(\omega) \pm B_{4}^{*}(\omega)\right] } \\
& \times\left[\sqrt{\eta} B_{1}(\omega) A_{4}(\omega)+\sqrt{\eta} B_{2}(\omega) A_{3}^{*}(-\omega) \pm B_{4}(\omega)\right) \\
& +\left[\sqrt{\eta} B_{2}^{*}(\omega) A_{4}(-\omega)+\sqrt{\eta} B_{1}^{*}(\omega) A_{3}^{*}(\omega) \pm B_{3}^{*}(\omega)\right] \\
& \times\left[\sqrt{\eta} B_{1}(\omega) A_{3}(\omega)+\sqrt{\eta} B_{2}(\omega) A_{4}^{*}(-\omega) \pm B_{3}(\omega)\right] \\
& +B_{4}^{*}(\omega) B_{4}(\omega)+B_{3}^{*}(\omega) B_{3}(\omega), \\
D_{3}= & {\left[\sqrt{\eta} B_{2}^{*}(\omega) A_{5}(-\omega)+\sqrt{\eta} B_{1}^{*}(\omega) A_{6}^{*}(\omega) \pm B_{6}^{*}(\omega)\right] } \\
& \times\left[\sqrt{\eta} B_{1}(\omega) A_{6}(\omega)+\sqrt{\eta} B_{2}(\omega) A_{5}^{*}(-\omega) \pm B_{6}(\omega)\right] \\
& +\left[\sqrt{\eta} B_{2}^{*}(\omega) A_{6}(-\omega)+\sqrt{\eta} B_{1}^{*}(\omega) A_{5}^{*}(\omega) \pm B_{5}^{*}(\omega)\right] \\
& \times\left[\sqrt{\eta} B_{1}(\omega) A_{5}(\omega)+\sqrt{\eta} B_{2}(\omega) A_{6}^{*}(-\omega) \pm B_{5}(\omega)\right) \\
& +B_{6}^{*}(\omega) B_{6}(\omega)+B_{5}^{*}(\omega) B_{5}(\omega), \\
D_{4}= & (1-\eta)\left(B_{2}^{*}(\omega) B_{2}(\omega)+B_{1}^{*}(\omega) B_{1}(\omega)\right),
\end{aligned}
$$




$$
\begin{aligned}
F_{1}= & {\left[\sqrt{\eta} B_{2}^{*}(\omega) A_{1}(-\omega)+\sqrt{\eta} B_{1}^{*}(\omega) A_{2}^{*}(\omega) \pm B_{2}^{*}(\omega)\right] } \\
& \times\left[\sqrt{\eta} B_{1}(\omega) A_{2}(\omega)+\sqrt{\eta} B_{2}(\omega) A_{1}^{*}(-\omega) \pm B_{2}(\omega)\right], \\
F_{2}= & {\left[\sqrt{\eta} B_{2}^{*}(\omega) A_{3}(-\omega)+\sqrt{\eta} B_{1}^{*}(\omega) A_{4}^{*}(\omega) \pm B_{4}^{*}(\omega)\right] } \\
& \times\left[\sqrt{\eta} B_{1}(\omega) A_{4}(\omega)+\sqrt{\eta} B_{2}(\omega) A_{3}^{*}(-\omega) \pm B_{4}(\omega)\right] \\
& +B_{4}^{*}(\omega) B_{4}(\omega), \\
F_{3}= & {\left[\sqrt{\eta} B_{2}^{*}(\omega) A_{5}(-\omega)+\sqrt{\eta} B_{1}^{*}(\omega) A_{6}^{*}(\omega) \pm B_{6}^{*}(\omega)\right] } \\
& \times\left[\sqrt{\eta} B_{1}(\omega) A_{6}(\omega)+\sqrt{\eta} B_{2}(\omega) A_{5}^{*}(-\omega) \pm B_{6}(\omega)\right] \\
& +B_{6}^{*}(\omega) B_{6}(-\omega), \\
F_{4}= & (1-\eta) B_{2}^{*}(\omega) B_{2}(-\omega) .
\end{aligned}
$$

Then $\left\langle\left(\Delta N_{\eta,+}-\Delta N_{\eta,-}\right)^{2}\right\rangle_{H_{j}}$ can be calculated as

$$
\begin{aligned}
\left\langle\left(\Delta N_{\eta,+}-\Delta N_{\eta,-}\right)^{2}\right\rangle_{H_{j}}= & \left\langle N_{\eta,+}\right\rangle_{H_{j}}\left(\left\langle N_{\eta,+}\right\rangle_{H_{j}}+1\right)+\left\langle N_{\eta,-}\right\rangle_{H_{j}}\left(\left\langle N_{\eta,-}\right\rangle_{H_{j}}\right. \\
& +1)-\frac{\left(\left\langle d_{\eta, \mathrm{o}}^{\dagger} d_{\eta, \mathrm{o}}\right\rangle_{H_{j}}-\left\langle d_{\mathrm{o}}^{\dagger} d_{\mathrm{o}}\right\rangle\right)^{2}}{2},
\end{aligned}
$$

with $\left\langle d_{\mathrm{o}}^{\dagger} d_{\mathrm{o}}\right\rangle$ and $\left\langle d_{\eta, \mathrm{o}}^{\dagger} d_{\eta, \mathrm{o}}\right\rangle_{H_{j}}$ in Eq.(B.11) being

$$
\begin{aligned}
\left\langle d_{\mathrm{o}}^{\dagger} d_{\mathrm{o}}\right\rangle= & \left(B_{2}^{*}(\omega) B_{2}(\omega)+B_{1}^{*}(\omega) B_{1}(\omega)\right) \bar{n}_{\mathrm{w}}^{T}+\left(B_{4}^{*}(\omega) B_{4}(\omega)+\right. \\
& \left.B_{3}^{*}(\omega) B_{3}(\omega)\right) \bar{n}_{\mathrm{o}}^{T}+\left(B_{6}^{*}(\omega) B_{6}(\omega)+B_{5}^{*}(\omega) B_{5}(\omega) \bar{n}_{\mathrm{b}}^{T}+\right. \\
& B_{2}^{*}(\omega) B_{2}(\omega)+B_{4}^{*}(\omega) B_{4}(\omega)+B_{6}^{*}(\omega) B_{6}(\omega) \\
\left\langle d_{\eta, \mathrm{o}}^{\dagger} d_{\eta, \mathrm{o}}\right\rangle_{H_{1}}= & K_{1} \bar{n}_{\mathrm{w}}^{T}+K_{2} \bar{n}_{\mathrm{o}}^{T}+K_{3} \bar{n}_{\mathrm{b}}^{T}+K_{4} \bar{n}_{B}^{T}+\sum_{i=1}^{4} T_{i} \\
\left\langle d_{\eta, \mathrm{o}}^{\dagger} d_{\eta, \mathrm{o}}\right\rangle_{H_{0}}= & \left\langle d_{\eta, \mathrm{o}}^{\dagger} d_{\eta, \mathrm{o}}\right\rangle_{H_{1}}(\eta \rightarrow 0)
\end{aligned}
$$

where the coefficient of Eq. (B.13) is

$$
\begin{aligned}
K_{1}=\eta[ & \left(B_{2}^{*}(\omega) A_{1}(-\omega)+B_{1}^{*}(\omega) A_{2}^{*}(\omega)\right)\left(B_{1}(\omega) A_{2}(\omega)+B_{2}(\omega) A_{1}^{*}(-\omega)\right)+ \\
& \left.\left(B_{2}^{*}(\omega) A_{2}(-\omega)+B_{1}^{*}(\omega) A_{1}^{*}(\omega)\right)\left(B_{1}(\omega) A_{1}(\omega)+B_{2}(\omega) A_{2}^{*}(-\omega)\right)\right],
\end{aligned}
$$




$$
\begin{aligned}
& K_{2}=\eta\left[\left(B_{2}^{*}(\omega) A_{3}(-\omega)+B_{1}^{*}(\omega) A_{4}^{*}(\omega)\right)\left(B_{1}(\omega) A_{4}(\omega)+B_{2}(\omega) A_{3}^{*}(-\omega)\right)+\right. \\
& \left.\left(B_{2}^{*}(\omega) A_{4}(-\omega)+B_{1}^{*}(\omega) A_{3}^{*}(\omega)\right)\left(B_{1}(\omega) A_{3}(\omega)+B_{2}(\omega) A_{4}^{*}(-\omega)\right)\right]+ \\
& B_{4}^{*}(\omega) B_{4}(\omega)+B_{3}^{*}(\omega) B_{3}(\omega) \text {, } \\
& K_{3}=\eta\left[\left(B_{2}^{*}(\omega) A_{5}(-\omega)+B_{1}^{*}(\omega) A_{6}^{*}(\omega)\right)\left(B_{1}(\omega) A_{6}(\omega)+B_{2}(\omega) A_{5}^{*}(-\omega)\right)+\right. \\
& \left.\left(B_{2}^{*}(\omega) A_{6}(-\omega)+B_{1}^{*}(\omega) A_{5}^{*}(\omega)\right)\left(B_{1}(\omega) A_{5}(\omega)+B_{2}(\omega) A_{6}^{*}(-\omega)\right)\right]+ \\
& B_{6}^{*}(\omega) B_{6}(\omega)+B_{5}^{*}(\omega) B_{5}(\omega), \\
& K_{4}=(1-\eta)\left[B_{2}^{*}(\omega) B_{2}(\omega)+B_{1}^{*}(\omega) B_{1}(\omega)\right] \text {, } \\
& T_{1}=\eta\left[\left(B_{2}^{*}(\omega) A_{1}(-\omega)+B_{1}^{*}(\omega) A_{2}^{*}(\omega)\right)\left(B_{1}(\omega) A_{2}(\omega)+B_{2}(\omega) A_{1}^{*}(-\omega)\right)\right] \\
& T_{2}=\eta\left[\left(B_{2}^{*}(\omega) A_{3}(-\omega)+B_{1}^{*}(\omega) A_{4}^{*}(\omega)\right)\left(B_{1}(\omega) A_{4}(\omega)+B_{2}(\omega) A_{3}^{*}(-\omega)\right)\right] \\
& +B_{4}^{*}(\omega) B_{4}(\omega) \\
& T_{3}=\eta\left[\left(B_{2}^{*}(\omega) A_{5}(-\omega)+B_{1}^{*}(\omega) A_{6}^{*}(\omega)\right)\left(B_{1}(\omega) A_{6}(\omega)+B_{2}(\omega) A_{5}^{*}(-\omega)\right)\right] \\
& +B_{6}^{*}(\omega) B_{6}(\omega), \\
& T_{4}=(1-\eta) B_{2}^{*}(\omega) B_{2}(\omega) \\
& * \text { and * }
\end{aligned}
$$

\section{References}

\section{References}

[1] S. Lloyd, Science 321 (2008) 1463-1465.

[2] J. H. Shapiro, Phys. Rev. A 80 (2009) 022320.

[3] S. Zhang, X. Zou, J. Shi, J. Guo, G. Guo, Phys. Rev. A 90 (2014) 052308.

[4] Z. Zhang, S. Mouradian, F. N. C. Wong, J. H. Shapiro, Phys. Rev. Lett. 114 (2015) 110506.

[5] S. Zhang, J. Guo, W. Bao, J. Shi, C. Jin, X. Zou, G. Guo, Phys. Rev. A 89 (2014) 062309. 
[6] S.-H. Tan, B. I. Erkmen, V. Giovannetti, S. Guha, S. Lloyd, L. Maccone, S. Pirandola, J. H. Shapiro, Phys. Rev. Lett. 101 (2008) 253601.

[7] S. Guha, B. I. Erkmen, Phys. Rev. A 80 (2009) 052310.

[8] J. H. Shapiro, S. Lloyd, New Journal of Physics 11 (2009) 063045.

[9] E. D. Lopaeva, I. Ruo Berchera, I. P. Degiovanni, S. Olivares, G. Brida, M. Genovese, Phys. Rev. Lett. 110 (2013) 153603.

[10] G. Wang, L. Huang, Y.-C. Lai, C. Grebogi, Phys. Rev. Lett. 112 (2014) 110406.

[11] B. Rogers, M. Paternostro, G. M. Palma, G. De Chiara, Phys. Rev. A 86 (2012) 042323.

[12] M. C. Kuzyk, S. J. van Enk, H. Wang, Phys. Rev. A 88 (2013) 062341.

[13] A. Farace, F. Ciccarello, R. Fazio, V. Giovannetti, Phys. Rev. A 89 (2014) 022335.

[14] Z. J. Deng, X.-B. Yan, Y.-D. Wang, C.-W. Wu, Phys. Rev. A 93 (2016) 033842 .

[15] R.-X. Chen, L.-T. Shen, S.-B. Zheng, Phys. Rev. A 91 (2015) 022326.

[16] A. Xuereb, M. Barbieri, M. Paternostro, Phys. Rev. A 86 (2012) 013809.

[17] Y. Ma, S. L. Danilishin, C. Zhao, H. Miao, W. Z. Korth, Y. Chen, R. L. Ward, D. G. Blair, Phys. Rev. Lett. 113 (2014) 151102.

[18] B. Abbott, R. Abbott, R. Adhikari, P. Ajith, B. Allen, G. Allen, R. Amin, S. B. Anderson, W. G. Anderson, M. A. Arain, New Journal of Physics 11 (2009) 073032.

[19] K. Zhang, F. Bariani, Y. Dong, W. Zhang, P. Meystre, Phys. Rev. Lett. 114 (2015) 113601.

[20] W.-Z. Zhang, J. Cheng, J.-Y. Liu, L. Zhou, Phys. Rev. A 91 (2015) 063836.

[21] T. Yousif, W. Zhou, L. Zhou, J. of Mod. Opt. 61 (2014) 1180-1186. 
[22] W. Li, C. Li, H. Song, J. of Phys. B 48 (2015) 035503.

[23] F. Marquardt, A. Clerk, S. Girvin, J. of Mod. Opt. 55 (2008) 3329-3338.

[24] W.-Z. Zhang, J. Cheng, W.-D. Li, L. Zhou, Phys. Rev. A 93 (2016) 063853.

[25] H. Xiong, L.-G. Si, X.-Y. L, X. Yang, Y. Wu, Annals of Physics 349 (2014) $43-54$.

[26] S. Barzanjeh, S. Guha, C. Weedbrook, D. Vitali, J. H. Shapiro, S. Pirandola, Phys. Rev. Lett. 114 (2015) 080503.

[27] R. Xiao, G. Pan, L. Zhou, The Eur. Phys. J. D 67 (2013) 1-7.

[28] J. Cheng, W. Z. Zhang, L. Zhou, W. Zhang, Scientific. Reports. 6 (2015).

[29] J. Cheng, Y. Han, Q. zhi An, L. Zhou, Annals of Physics 354 (2015) 590 -603 .

[30] C. H. Bai, D. Y. Wang, H. F. Wang, A. D. Zhu, S. Zhang, Scientific. Reports. 6 (2016).

[31] Q. Mu, X. Zhao, T. Yu, Phys. Rev. A 94 (2016) 012334.

[32] G. Pan, R. Xiao, L. Zhou, Int. J. of Theore. Phys. 55 (2016) 3697-3705.

[33] D. Vitali, P. Tombesi, M. J. Woolley, A. C. Doherty, G. J. Milburn, Phys. Rev. A 76 (2007) 042336.

[34] S. Huang, G. S. Agarwal, Phys. Rev. A 80 (2009) 033807.

[35] S. Barzanjeh, D. Vitali, P. Tombesi, G. J. Milburn, Phys. Rev. A 84 (2011) 042342.

[36] S. Barzanjeh, M. Abdi, G. J. Milburn, P. Tombesi, D. Vitali, Phys. Rev. Lett. 109 (2012) 130503.

[37] Y.-D. Wang, A. A. Clerk, Phys. Rev. Lett. 110 (2013) 253601.

[38] Y.-D. Wang, S. Chesi, A. A. Clerk, Phys. Rev. A 91 (2015) 013807.

[39] E. X. DeJesus, C. Kaufman, Phys. Rev. A 35 (1987) 5288-5290. 
[40] G. Adesso, A. Serafini, F. Illuminati, Phys. Rev. A 70 (2004) 022318.

[41] X.-Y. Lü, Y. Wu, J. R. Johansson, H. Jing, J. Zhang, F. Nori, Phys. Rev. Lett. 114 (2015) 093602.

[42] D.-Y. Wang, C.-H. Bai, H.-F. Wang, A.-D. Zhu, S. Zhang, Scientific Reports 6 (2016).

[43] D.-Y. Wang, C.-H. Bai, H.-F. Wang, A.-D. Zhu, S. Zhang, Scientific Reports 6 (2016).

[44] Z. Zhang, M. Tengner, T. Zhong, F. N. C. Wong, J. H. Shapiro, Phys. Rev. Lett. 111 (2013) 010501. 